\title{
Shear-Induced Viscosity Change of Aqueous Polymethacrylic Acid Solution
}

\author{
Seiji Ohoya, ${ }^{\dagger}$ Shigeo Hashiya, Kyoji Tsubakiyama, and Togoro Matsuo \\ Faculty of Engineering, Fukui University, 3-9-1 Bunkyo, Fukui 910-8507, Japan
}

(Received June 2, 1999)

\begin{abstract}
Rheopectic behavior of the aqueous polymethacrylic acid (PMA) solution was investigated with a Zimm-Crothers type viscometer. Measurements were performed for the solutions of PMA ranging in molecular weight from $6 \times 10^{5}$ to $16 \times 10^{5}$, at concentration of $0.05-5.0 \mathrm{wt} \%$ and shear rate of $0.5-7.0 \mathrm{~s}^{-1}$. The solution viscosities of high molecular weight PMA $\left(M_{V}>10 \times 10^{5}\right)$ increase linearly with the elapse of shearing time at early stage, reach a maximum and then fall off. Ratio of the maximum viscosity to the initial one, $\eta_{\max } / \eta_{\text {init }}$, decreases with decreasing the concentration of polymer and increasing the rate of shear, indicating that rheopexy is attributed to a competition between growth and disruption of the molecular clusters in the shear field. The influences of four additives $(\mathrm{HCl}, \mathrm{NaOH}, \mathrm{NaCl}$, and urea) on rheopexy were examined; it was found that, regardless of the additive species, there is a correlation between rheopexy and the initial rate of shear. These results suggest that a particular balance of hydrophilic and hydrophobic bonding abilities of PMA molecule is essential for the occurrence of rheopexy in the solution.

KEY WORDS Polymethacrylic Acid Solution / Rotating Cylinder Viscometer / Rheopexy / ShearInduced Viscosity Change / Time-Dependent Viscosity Change /
\end{abstract}

Although thixotropy has been observed in various polymer solutions, rheopexy and antithixotropy seem to be comparatively rare phenomena. ${ }^{1-9}$ Thixotropy refers to a decrease in the solution viscosity by a shear stress, while antithixotropy (or negative thixotropy) denotes an increase in viscosity. Both the phenomena are reversible, and it is possible to return the system to the original state by removing the stress. Certain thixotropic systems of non-globular particles show a peculiar behavior, called rheopexy, and this is the case when gelation of a solution is facilitated by such mechanical force as a shear stress.

The anomalous rheological behavior of dilute polymer solutions, indicated by the time-dependent increase in viscosity at constant shear rate $\mathrm{e}^{2-3,5,10-12}$ and by the sheardependent viscosity increase (shear thickening) 6 $^{6-10,13-16}$ have been investigated using either a capillary viscometer or a rotating cylinder one. These rheopectic phenomena are attributed to the development of networks between the particles throughout the solution. Rheopexy have been observed more frequently for the concentrated dispersions of surfactants ${ }^{11,13}$ and inorganic substances such as silica ${ }^{1214-16}$ than for the dilute polymer solutions. ${ }^{6-10}$ Antithixotropy has been observed also in several polymer solutions. ${ }^{1,17-18}$ As an explanation for these phenomena, it has been proposed that the field of shear builds up a network through the intermolecular force but severs concurrently it also, and the former effect predominates in these systems. The mechanism of formation of the structure, however, seems to be still not completely elucidated.

Polymethacrylic acid (PMA) which has the hydrophobic methyl side chain shows some peculiar properties differing from those of usual polyelectrolytes. ${ }^{1,17,18}$ Eliassaf and co-workers ${ }^{1}$ have described the so-called negative thixotropy as an example of such peculiarities. That is, a $5 \%$ solution of PMA gives a 350 fold increase in viscosity when sheared at $10 \mathrm{~s}^{-1}$ for $1 \mathrm{~min}$; the transforma-

\footnotetext{
${ }^{\dagger}$ To whom all correspondence should be addressed.
}

tion is quite reversible, and the system returns to its original state in $1 \mathrm{~h}$ or so after the cessation of stirring. In our preliminary work, ${ }^{5}$ we investigated the behavior of PMA solutions and found that the time-dependent change in viscosity was irreversible, though the measurements were carried out in a relatively low concentration range.

It appears to admit of little doubt that rheopexy reflects a transition of chain conformation in the field of flow. The conformational change of the PMA chain is governed by dissociation of the carboxyl groups, ${ }^{19,20}$ and by the intermolecular and intramolecular hydrophobic $^{17,19-27}$ and/or hydrophilic ${ }^{1,28-32}$ bonding abilities. Concentration and molecular weight of the polymer and the rate of shear may also be the common and essential factors to affect rheopexy.

In the present paper, a series of experiments concerned with the shear-induced viscosity change in the solutions of PMA have been made to elucidate the origin of the anomalous behaviors described above. In practice, changes of viscosity in the aqueous solutions of PMA, polyacrylic acid (PAA), and polyvinyl pyrolidone (PVP) with the elapse of time were measured by using a rotating cylinder viscometer. For PMA solution, the timedependent changes in viscosity were examined in detail by changing concentration and molecular weight of polymer, and the rate of shear. The influences of urea and several electrolytes on the time-dependence of viscosity were also examined.

\section{EXPERIMENTAL}

\section{Materials}

Methacrylic acid distilled fractionally was polymerized in bulk at $50^{\circ} \mathrm{C}$ for $3 \mathrm{~h}$ by the degassed and sealed tube method using azoisobutyronitrile as an initiator. ${ }^{33}$ Three PMA samples of differing molecular weights (PMA-I, II, III ) were obtained by changing the initiator concentration. The polymers were reprecipitated from methanol solution into ether four times, dissolved in the 
deionized water, and then freeze-dried. PAA was prepared by polymerization of the monomer in benzene initiated with benzoyl peroxide. The polymer was washed several times alternately with ether and methanol, extracted with ether, dissolved in dioxane, and freezedried. PVP was a commercial product (Nakalai Tesque) and used without further purification. Molecular weights of these polymers estimated by using the viscosity-molecular weight relationships ${ }^{34}$ are summarized in Table I. Water was passed through an ionexchange resin column (ranging in electric conductivity from 0.6 to $1.0 \times 10^{-6} \mho$ ) and distilled just before use.

\section{Viscosity Measurements}

A Zimm-Crothers viscometer was constructed to examine the time-dependent change in viscosity in the low shear rate region. The viscometer is the same type as that modified by Sloniewsky et al., ${ }^{35}$ except for its stator and rotor being made out of a sample tube for NMR. The stator has inner diameter and height of 8.7 and $70 \mathrm{~mm}$, and outer diameter and height of the rotor are 8.0 and $60 \mathrm{~mm}$, respectively. The rotor was floated itself in the center of the stator so as to let its lip meet the meniscus by the surface tension of the solution and by the buoyancy acting on the rotor. The same rotor was sunk reverse near bottom of the stator in the Cartesian-diver method. Revolution velocity of the external magnet that drives the rotor, which has an aluminium ring at the bottom, was varied to controll the rate of shear. The viscometer was kept at $30 \pm 0.01{ }^{\circ} \mathrm{C}$ during the measurements.

According to Berry, ${ }^{36}$ relative viscosity $\eta_{\text {rel }}$ is given as follows ;

$$
\begin{aligned}
\eta_{\text {rel }} & =\eta / \eta_{0} \\
& =\left(1 / \Omega_{\mathrm{r}}-1 / \Omega_{\mathrm{m}}\right) /\left(1 / \Omega_{\mathrm{r}, 0}-1 / \Omega_{\mathrm{m}}\right)
\end{aligned}
$$

where $\eta$ and $\eta_{0}$ are viscosities of the solution and solvent; $\Omega_{\mathrm{r}}, \Omega_{\mathrm{r}, 0}$, and $\Omega_{\mathrm{m}}$ stand for revolution velocities of the rotor in the solution, that in the solvent, and of the magnet in rpm, respectively.

The polymer solutions were prepared just before measurements. They were centrifuged at $2 \times 10^{4} \mathrm{rpm}$ for $1 \mathrm{~h}$ and then filtered through a fine sintered glass disc. For preparation of the solutions containing an additive such as $\mathrm{HCl}, \mathrm{NaOH}, \mathrm{NaCl}$, and urea, the aqueous solution of PMA was mixed with equivolume of the stock solution of the additives. In order to check the hysteresis effect due to preparation process, a $1 \mathrm{wt} \%$ solution of PMA was relaxed overnight in the viscometer. No appreciable difference in viscosity, however, was observed before and after the relaxation. Hence, after allowing the solution to stand for $30 \mathrm{~min}$ at $30^{\circ} \mathrm{C}$ in the viscometer, each run was started. The precision of measurement was tested with water and the capability was examined by comparing the specific viscosities of aq PVP solution (Newtonian fluid) measured by a Ubbelohde viscometer with those measured by the rotating cylinder viscometer; the accuracy of measurement lay within $\pm 0.05 \%$ and the difference in viscosties with both viscometers was $\pm 1 \%$.
Table I. Molecular weight of polymers

\begin{tabular}{rcc}
\hline Polymer & $\frac{[\eta]}{\mathrm{dL} \mathrm{g}^{-1}}$ & $\frac{M_{v}}{\times 10^{5}}$ \\
\hline PMA- I & $0.82_{8}$ & $15.7^{\mathrm{a}}$ \\
- II & $0.70_{2}$ & 11.3 \\
- III & $0.50_{5}$ & 5.9 \\
PAA & $0.75_{0}$ & $7.8^{\mathrm{b}}$ \\
PVP & $0.57_{5}$ & $13.3^{\mathrm{c}}$
\end{tabular}

${ }^{\mathrm{a}}[\eta]=6.6 \times 10^{-4} M_{w}{ }^{0.50}$ in aq $\mathrm{HCl}(0.002 \mathrm{M})$ at $30^{\circ} \mathrm{C} .{ }^{\mathrm{b}}[\eta]=7.6 \times$ $10^{-4} M_{w}^{0.50}$ in dioxane at $30^{\circ} \mathrm{C} .{ }^{\mathrm{c}}[\eta]=6.76 \times 10^{-4} M_{w}{ }^{0.55}$ in water at $25^{\circ} \mathrm{C}$.

\section{Light Scattering Measurements}

Intensities of the light scattered from the aqueous PMA-II solution were measured at scattering angles of 45,90 , and $135^{\circ}$ to examine whether the timedependent change in the scattered light intensity was caused with stirring the solution. A Brice type light scattering photometer (Shimadzu PG-21) with the incident beam of $546 \mathrm{~nm}$ was employed. The cuvette was a cylindrical cell having inner diameter of $38 \mathrm{~mm}$ and height of $50 \mathrm{~mm}$, consequently volume of the solution was about $60 \mathrm{~mL}$, and mantled with a water jacket kept at $30^{\circ} \mathrm{C}$. An iron tip covered with glass was put in the cell and rotated magnetically to stir the solution. Before the measurement the solution was centrifuged at $2 \times 10^{4} \mathrm{rpm}$ for 1 $\mathrm{h}$ and passed through a membrane filter into the cell.

\section{RESULTS}

Time-Dependent Change in Viscosity of Aqueous Polymer Solutions

Relative viscosity, $\eta_{\text {rel }}$, is plotted as ordinate and the square root of shearing time, $t^{1 / 2}$, as abscissa in Figure 1. Curves $1-3$ represent three runs for a $1.4 \mathrm{wt} \%$ aqueous solution of PMA- $I$ at $30^{\circ} \mathrm{C}$, in which either the purification method or the rotor position was changed. The results for the solutions of PAA and PVP are also shown in the Figure for comparison (curves $4-5$ ).

Curve 1 was obtained for the solution purified by filtration alone. The dotted region indicates the rest period of the shearing. Even though the shearing was discontinued overnight, it seemed unlikely that the system should return to the original state. The solution centrifuged prior to filtration, as shown with curve 2, gives lower $\eta_{\text {rel }}$ and more rapid arrival at a maximum compared with the uncentrifuged one (curve 1). It should be noticed that reproducibility of the data is better than that of curve 1 . Thus, subsequent measurements were decided to carry out all for the centrifuged solutions. Curve 3 refers to an experiment in which the rotor was sunk into the solution as a Cartesian-diver to avoid any meniscus effect. The result obtained is virtually analogous to that of run 2 , except that the reproducibility is relatively poor. Thus, in all the cases of PMA-II, $\eta_{\text {rel }}$ increases almost linearly with $t^{1 / 2}$ at the early stage, reaches a maximum and then falls off gradually. In the solutions of PAA and PVP, on the other hand, the values of $\eta_{\text {rel }}$ either remain constant or slightly decrease with the elapse of time (curves 4 and 5 ). 


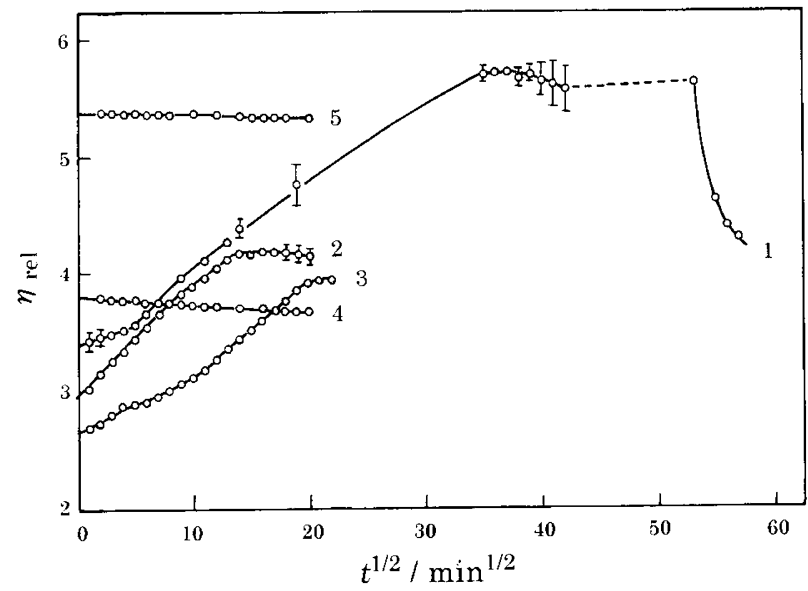

Figure 1. Time-dependent change of viscosity in aqueous $1.4 \mathrm{wt}$ $\%$ polymer solutions at $\Omega_{\mathrm{m}}=200 \mathrm{rpm}$ and $30^{\circ} \mathrm{C}$. Runs $1-3$ refer to PMA- $\Pi$, run 4 to PAA and run 5 to PVP. The polymer solutions were purified with (runs 2-5) and without (run 1) centrifugation. Run 3 is the data measured with the Cartesian-diver rotor.

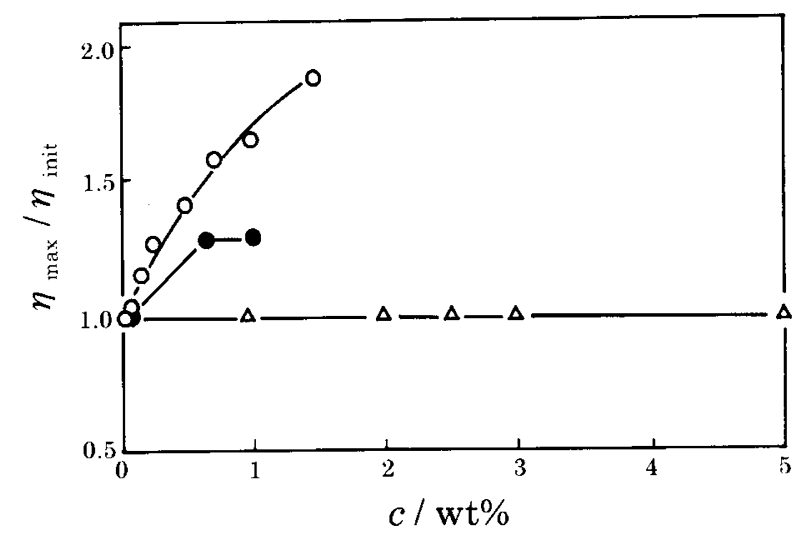

Figure 2. Change in ratio of the maximum relative viscosity to the initial one $\eta_{\max } / \eta_{\text {init }}$ with polymer concentration at $\Omega_{\mathrm{m}}=200$ rpm and $30^{\circ} \mathrm{C}$ : PMA-I $(\bigcirc)$, PMA-I $(\bigcirc)$, and PMA-II $(\triangle)$.

\section{Effects of PMA Concentration and Molecular Weight and} Rate of Shear on Rheopexy

Changes in viscosity with time were examined in the concentration range $c=0.05-5 \mathrm{wt} \%$ for three PMA samples ranging in molecular weight from $59 \times 10^{4}$ to $157 \times$ $10^{4}$. The ratio of $\eta_{\max }$ to $\eta_{\text {init }}$ is plotted against $c$ in Figure 2 , where $\eta_{\max }$ is the maximum value of $\eta_{\text {rel }}$ in the $\eta_{\text {rel }} v s$. $t^{1 / 2}$ curve (Figure 1) and $\eta_{\text {init }}$ is estimated by extrapolation of $\eta_{\text {rel }}$ to time zero. In the cases of higher molecular weight polymers (PMA-I and II), the time-dependent changes in viscosity were observed above $c=0.1 \mathrm{wt} \%$ and the ratio $\eta_{\max } / \eta_{\text {init }}$ increase with increasing the polymer concentration. No time-dependent behavior, on the contrary, was observed on the lowest molecular weight one (PMA-III), even though its concentration was raised up to $5 \mathrm{wt} \%$.

In order to describe a relationship between the rates of shear at start (init) and at equilibrium (max), $\gamma_{\text {init }}$ and $\gamma_{\max }$ were calculated on the data of PMA- $I$ in Figure 2. The relative viscosity determined by the rotating cylinder viscometer is expressed as

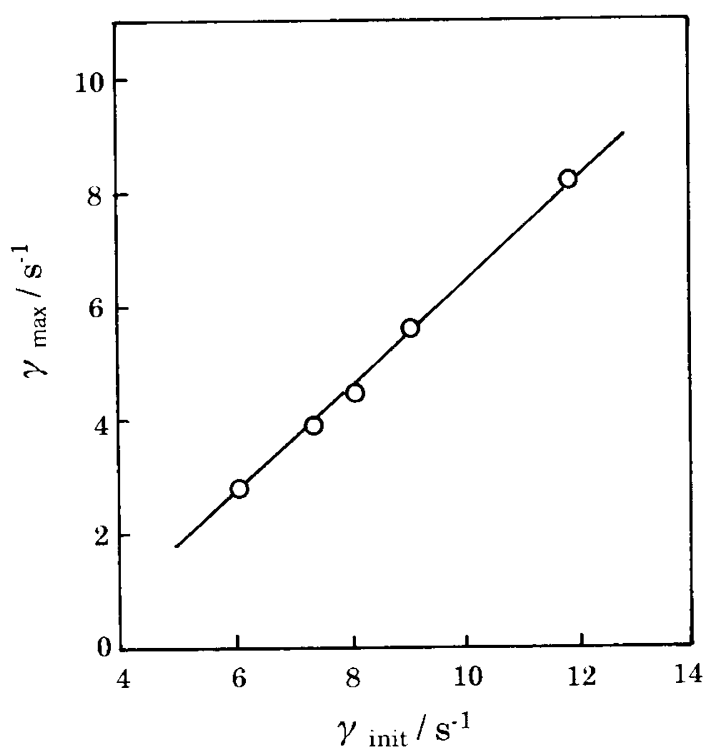

Figure 3. Relation between the rate of shear at maximum viscosity, $\gamma_{\max }$, and the initial shear rates $\gamma_{\text {init }}$ at $\Omega_{\mathrm{m}}=200 \mathrm{rpm}$ and $30^{\circ} \mathrm{C}$ for PMA- II .

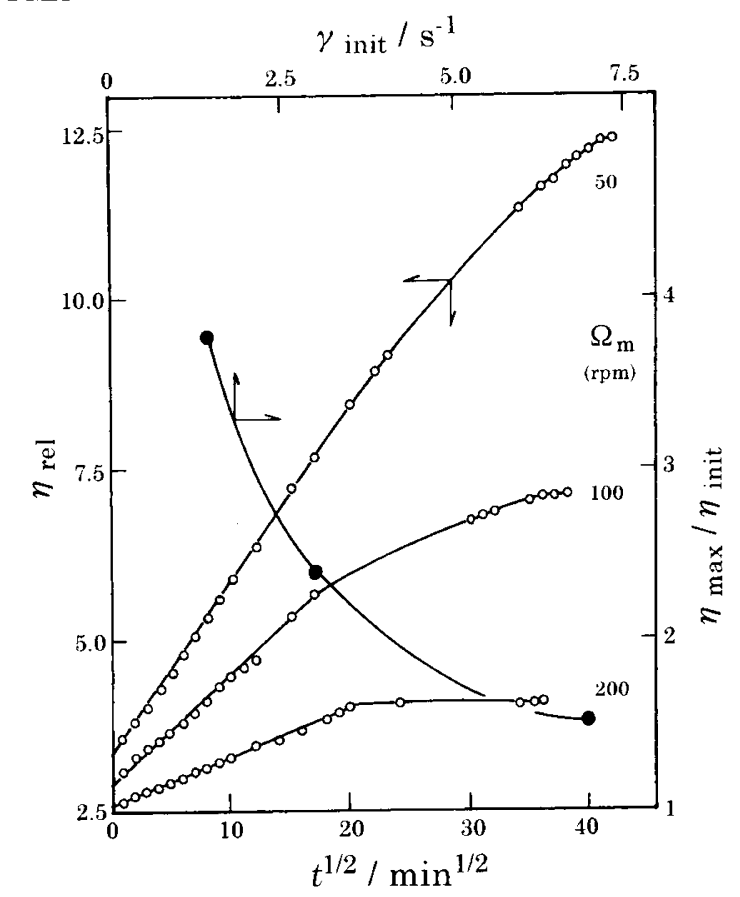

Figure 4. Time-dependent viscosity change in aqueous solution of PMA- II : effect of revolution velocity of the magnet $(\bigcirc)$ and relation between $\eta_{\max } / \eta_{\text {init }}$ and the initial rate of shear $\gamma_{\text {init }}(\mathbf{O})$ at $c=$ $1.04 \mathrm{wt} \%$ and $30^{\circ} \mathrm{C}$.

$$
\eta_{\text {rel }}=\eta / \eta_{0}=\left(t-t_{\mathrm{m}}\right) /\left(t_{0}-t_{\mathrm{m}}\right)
$$

where $t_{\mathrm{m}}$ is the period of revolution of the magnet in second, and $t$ and $t_{0}$ refer to the solution and the solvent, respectively. The average shear rate $<\gamma>$ is given by

$$
\langle\gamma\rangle=(\pi / t)\left[\left(R_{2}+R_{1}\right) /\left(R_{2}-R_{1}\right)\right] f\left(R_{2}, R_{1}\right)
$$

here $R_{2}$ and $R_{1}$ are radii of the stator and the rotor $\left(R_{2}>\right.$ $\left.R_{1}\right)$, and $f\left(R_{2}, R_{1}\right)=8\left(R_{2} / R_{1}\right)^{2} \ln \left(R_{2} / R_{1}\right) /\left(R_{2} / R_{1}+1\right)^{3}\left(R_{2} / R_{1}\right.$ $-1)$ is 0.994 for this apparatus. Relation between $\gamma_{\text {init }}$ and $\gamma_{\max }$ calculated from $\eta_{\text {init }}$ and $\eta_{\max }$ in terms of (2) and 
(3) is given in Figure 3 . The presence of a substantially linear relation between $\gamma_{\max }$ and $\gamma_{\text {init }}$ is revealed.

Figure 4 represents the effect of revolution velocities of the magnet on the time-dependent viscosity changes in $1 \mathrm{wt} \%$ solution of PMA-II. In the Figure, $\eta_{\max } / \eta_{\text {init }}$ is also plotted as a function of the initial rate of shear, $\gamma_{\text {init }}$. The increased shear rate tends to suppress the timedependent increase in $\eta_{\text {rel }}$ and to reduce an arrival time at the maximum, and $\eta_{\max } / \eta_{\text {init }}$ varies inversely with $\gamma_{\text {init }}$.

\section{Rheopexy in the Presence of Additives}

Effects of four additives ( $\mathrm{HCl}, \mathrm{NaOH}, \mathrm{NaCl}$, and urea) on the viscosity change were examined to clarify the rheopectic behavior. The experiments shown in this section were all carried out for a $1.45 \mathrm{wt} \%$ solution of PMA- $I$ at $\Omega_{\mathrm{m}}=200 \mathrm{rpm}$ and $30^{\circ} \mathrm{C}$.

Figure 5 shows the variation in relative viscosity observed in the presence of hydrochloric acid ranging in concentration from 0.001 to $0.2 \mathrm{~mol} \mathrm{~L}^{-1}$. As concentration of the acid is raised up to $0.05 \mathrm{~mol} \mathrm{~L}^{-1}$, both $\eta_{\text {init }}$ and $\eta_{\max }$ decreased rapidly, but increased again above this concentration. Reproducibility of the measurement becomes progressively poor beyond $0.2 \mathrm{~mol} \mathrm{~L}^{-1}$, until the polymer precipitates at $1 \mathrm{~mol} \mathrm{~L}^{-1}$.

Measurements similar to the above were made in the presence of sodium hydroxide of concentrations of 0.0017 $-0.17 \mathrm{~mol} \mathrm{~L}^{-1}$. The viscosity changes with time are given in Figure 6, where $i$ stands for the degree of neutralization in \%. The time-dependence of viscosity similar to that of the solution without the base was observed at low degree of neutralization, but the time-dependence disappeared at the neutralization degree of $4 \%$ or above.

The values of $\eta_{\text {init }}$ and $\eta_{\max } / \eta_{\text {init }}$ for the solutions containing sodium chloride $\left(0.001-0.25 \mathrm{~mol} \mathrm{~L}^{-1}\right)$ are plotted as a function of molar concentration of the salt in Figure $7 . \eta_{\text {init }}$ and $\eta_{\text {max }} / \eta_{\text {init }}$ decrease with increasing the salt content up to $0.2 \mathrm{~mol} \mathrm{~L}^{-1}$. Over this concentration, $\eta_{\max } / \eta_{\text {init }}$ tends to increase steeply until a gelation by the salting-out effect occurs, resulting in poor reproducibility.

Figure 8 represents the same plots as those in Figure 7 for the solutions containing urea whose content ranges 0.05 to $8.0 \mathrm{~mol} \mathrm{~L}^{-1} . \eta_{\max } / \eta_{\text {init }}$ and $\eta_{\text {init }}$ decreased with increasing the urea concentration and commenced to rise again at about $4.0 \mathrm{~mol} \mathrm{~L}^{-1}$, both quantities varying with almost a parallel tendency. Furthermore, it was found that urea delays the arrival time at $\eta_{\max }$, and that the time-dependent change in viscosity (rheopexy) is not observed in the region of $2.0-4.0 \mathrm{~mol} \mathrm{~L}^{-1}$ of urea.

\section{Light Scattering}

In Figure 9 are shown the time-dependent changes in the Rayleigh ratio, $\mathrm{R}_{90}$, and the dissymmetry, $Z\left(=R_{45} /\right.$ $R_{135}$ ), for 1.0 and $3.0 \mathrm{wt} \%$ solutions of PMA- II in water. It was observed that both the scattered intensity and the dissymmetry are increased by about $3 \%$, regardless of concentrations of PMA, if an agitation is applied to the solutions.

\section{DISCUSSION}

In the begining of discussion, we are confronted by a problem whether the time-dependent change in rotor

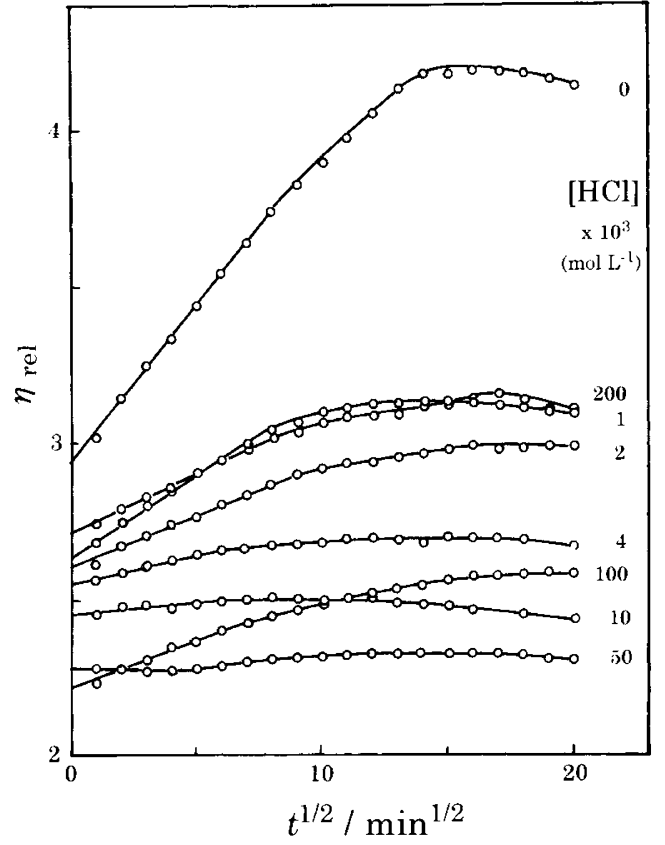

Figure 5. Time-dependent change of viscosity in aqueous PMA II solution containing hydrochloric acid at $\Omega_{\mathrm{m}}=200 \mathrm{rpm}, c=1.45$ wt $\%$, and $30^{\circ} \mathrm{C}$

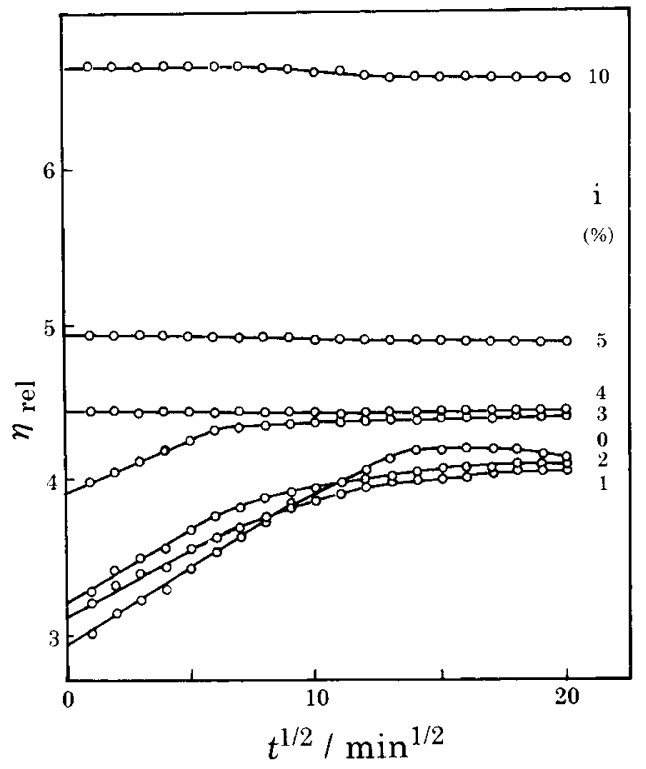

Figure 6. Time-dependent change of viscosity in aqueous PMAII solution containing sodium hydroxide at $\Omega_{\mathrm{m}}=200 \mathrm{rpm}, c=1.45$ wt $\%$, and $30^{\circ} \mathrm{C}$. Symbol $i$ denotes the degree of neutralization in $\%$.

speed is ascribed to a change in the solution state in bulk or to that in the meniscus state, because the revolution velocity can be varied by both ascriptions in the floating type rotor. In order to elucidate this question, a parallel experiment was made by using the floating type and the Cartesian-diver type rotors, of which the latter is taken as valid to avoid any surface effect. Two runs on PMA-II solution are in qualitatively agreement as seen from the curves 2 and 3 in Figure 1 . It may be thus concluded that the observed change in rotor speed, namely in viscosity, is caused essentially by the structural change throughout the bulk of the solution. It should be noted, however, that the floating type rotor gives some- 


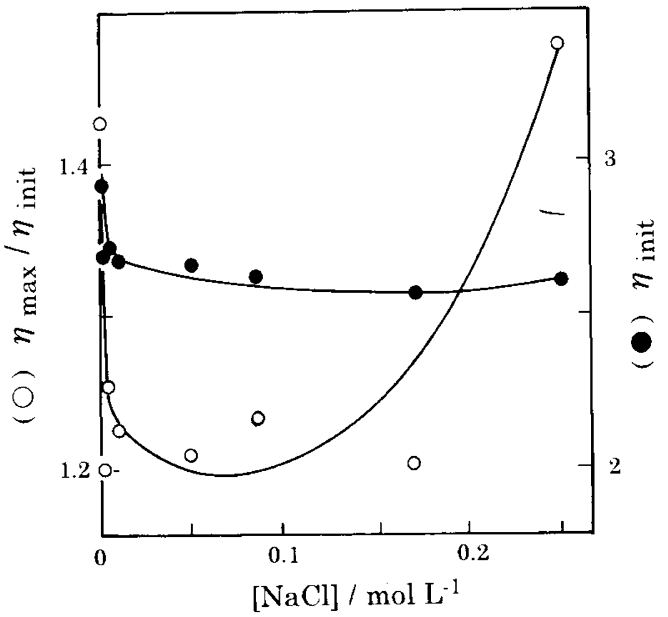

Figure 7. $\eta_{\text {init }}(\bigcirc)$ and $\eta_{\max } / \eta_{\text {init }}(\bigcirc)$ as a function of concentration of sodium chloride at $\Omega_{\mathrm{m}}=200 \mathrm{rpm}, c=1.45 \mathrm{wt} \%$, and $30^{\circ} \mathrm{C}$.

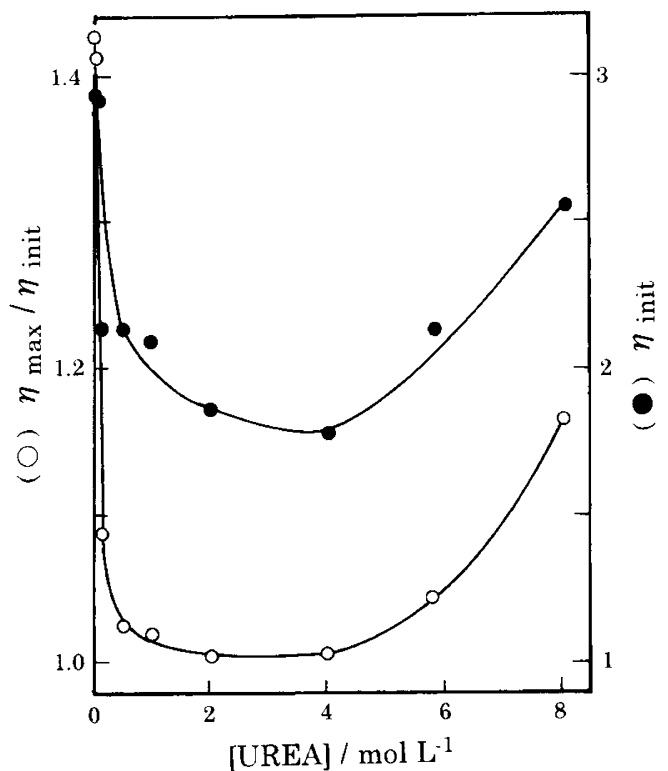

Figure 8. $\eta_{\text {init }}(O)$ and $\eta_{\max } / \eta_{\text {init }}(O)$ as a function of concetration of urea at $\Omega_{\mathrm{m}}=200 \mathrm{rpm}, c=1.45 \mathrm{wt} \%$, and $30^{\circ} \mathrm{C}$.

what higher viscosity than that given by the Cartesiandiver type, suggesting some additional transformation at the surface.

Another evidence for such a shear-induced structure formation is offered by a preliminary test on the light scattering measurements. That is to say, stirring of the solution bring about small but distinguishable increase in the scattered light intensity and its angular dissymmetry (Figure 9).

Neglecting the meniscus effect in the Zimm-Crothers viscometer, it turns out that the shear rate is almost inversely proportional to the relative viscosity at a constant rotational velocity of the magnet. Hence, in all the runs which show the time-dependent increase in viscosity as illustrated in Figures 1 and $4-6$, the rate of shear continues to go down to its minimum value corresponding to the maximum viscosity as shown in Figure 3.

A quantitative analysis on the contribution of the rate of shear to the time-dependent change in viscosity is very difficult owing to the above particular circumstance. The pronounced increase in $\eta_{\max } / \eta_{\text {init }}$ ratio with decreas-

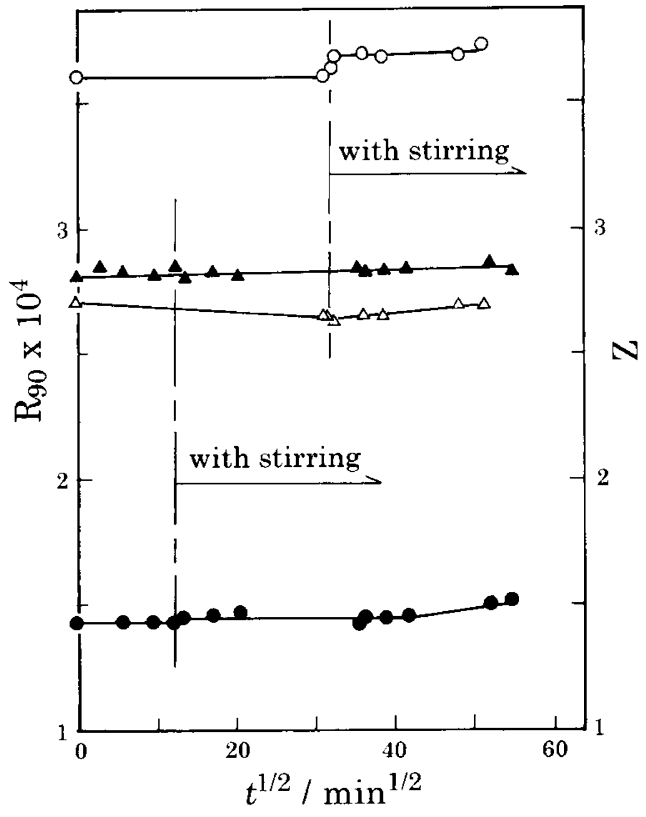

Figure 9. Time-dependent changes in the scattered light intensity $R_{90}(\bigcirc, \bigcirc)$ and the dissymmetry $Z(\triangle, \Delta)$ with and without stirring. Open and closed marks correspond to PMA- II concentrations of 3.0 and $1.0 \mathrm{wt} \%$, respectively. Wave length of the incident beam is $546 \mathrm{~nm}$ and temp. $30^{\circ} \mathrm{C}$.

ing the initial shear rate (Figure 4), however, indicate that there is a competitive construction and destruction of the structure in the solution. Thus the lower the initial shear rate, the more the structure is constructed and survived, and the resultant higher viscosity makes the rate of shear still lower, or vice versa. Moreover, it is suggested from Figure 1 that the structure induced mechanically may be too weak against the shearing force to grow up to a large scale; such a structure, therefore, seems to be referred to a small cluster of PMA molecules. Interaction between the clusters makes the disintegration predominant when their size and population enlarge to a certain extent, resulting in the decrease in $\eta_{\text {rel }}$ after passing the maximum. It is also pointed out that a thixotropic structure is formed in the rest period, ${ }^{37}$ and if so, the restart of revolution will lead to a decrease in viscosity. Unless the shearing is ceased, the formation of cluster could balance with its disruption to give a steady viscosity and the most system reaches equilibrium.

Besides the above considerations, two aspects of the results for viscometry of the aqueous solutions of PMA are important. First, the value of $\eta_{\text {rel }}$ does not revert to the initial one even though the shearing is interrupted overnight, as seen from the curve 1 in Figure 1 ; that is, the clusters once grown have been able to survive in the standing solution. From this fact the term "Rheopexy" is preferred for designation of the present phenomenon rather than "negative thixotropy", though both terms have been confusedly employed sometimes. The antithixotropic phenomenon, on the other hand, was found by Eliassaf et al. ${ }^{1}$ with the similar PMA solutions in 1955. They reported that a 5\% solution of PMA of molecular weight $8.6 \times 10^{5}$, being the concentration somewhat higher than that in this work, gave a great increase in viscosity on shearing at $10 \mathrm{~s}^{-1}$ and reverted to the original viscosity about $1 \mathrm{~h}$ after the cessation of 
shearing. An interrelation, however, should exist between their observation and the ours. Pondering over this, an observation made by Ono et al.$^{38}$ is fairly suggestive. They have examined the negatively thixotropic behavior for aqueous PMA solutions with a rotational viscometer at the constant rate of shear and found the induction period to be prolonged with decreasing the rate of shear.

The following interrelation may be presumed from such observations : in the solution of high concentration, the small clusters occur even at early stage and play a role of embryos or precursors for forming large but temporary networks; after ceasing the shearing, the large networks disintegrates, but the embryos live in the solution. It should be pertinent here, to emphasize that the pronounced antithixotropy was frequently witnessed during the treatment of the unpurified solution at an extremely high concentration; in addition, more increased viscosity was always observed in the solution uncentrifuged (for example, run-1 in Figure 1). These facts mean, therefore, that the micro gels existing in the solution may intensify both rheopexy and antithixotropy.

Another feature is that the rheopectic behavior appears at the very low polymer concentration of $0.1 \mathrm{wt} \%$ (Figure 2). Let us consider this critical concentration on the basis of the equivalent hydrodynamic sphere principle, since the clusters would be taken as formed through collision between PMA molecules. The Flory-Fox viscosity equation ${ }^{39}$ can be employed for this purpose by assuming that the radius of the equivalent sphere equals that of gyration, and the volume of the equivalent sphere, $V_{\mathrm{e}}$, is expressed as follows,

$$
\begin{aligned}
V_{\mathrm{e}} & =4 \pi<S^{2}>^{3 / 2} / 3 \\
& =4 \pi[\eta] M / 3 \Phi^{\prime} \\
& \doteqdot 4 \pi\left[\left(\ln \eta_{\text {rel }}\right) / c\right] M / 3 \Phi^{\prime}
\end{aligned}
$$

where $\left\langle S^{2}\right\rangle$ is the mean square radius of gyration, [ $\eta$ limiting viscosity number in $\mathrm{dL}^{-1}, \mathrm{M}$ molecular weight of polymer, $c$ concentration in $\mathrm{g} \mathrm{dL}^{-1}$, and $\Phi^{\prime}=6^{3 / 2}$ $\Phi$ (Flory's constant $\Phi=2.5 \times 10^{21}$ was used here). It is possible to evaluate the value of $V_{\mathrm{e}}$ if the value of $[\eta]$ is available. This procedure has been done according to Eliassaf et $a l .^{40}$ who estimated the value of $V_{\mathrm{e}}$ at the critical concentration of thermal gelation in PMA solution. In the case of polyelectrolyte such as PMA, however, the polymer dissociates progressively as its concentration is lowered. As the result, the charges enhanced on the polymer coil expand the coil itself tremendously at infinite dilution. It is, therefore, difficult to evaluate the correct $[\eta]$ value in a reliable way; even if the value of $[\eta]$ could be obtained in terms of an evaluation method, for instance, such as Fuoss's emperical relation ${ }^{41}\left(c / \eta_{\mathrm{sp}}=1\right.$ /

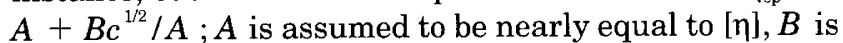
a constant involving the interaction between polyions, $\eta_{\mathrm{sp}}$ specific viscosity, and $c$ polymer concentration), it is somewhat questionable to use such a value for estimation of $V_{\mathrm{e}}$ at a finite concentration. Since the PMA concentration at which rheopexy appears is very low as described above, it is reasonable to assume that [ $\eta$ ] can be replaced by $\left(\ln \eta_{\text {re }}\right) / c$ in eq 4 . On applying the experimental value of 1.5 for $[\eta]$ at the critical concentration $(c=$ $0.1 \mathrm{~g} \mathrm{dL}^{-1}$ and $\gamma_{\text {init }} \doteqdot 10 \mathrm{~s}^{-1}$ ) and $11.3 \times 10^{5}$ for $\mathrm{M}$, the

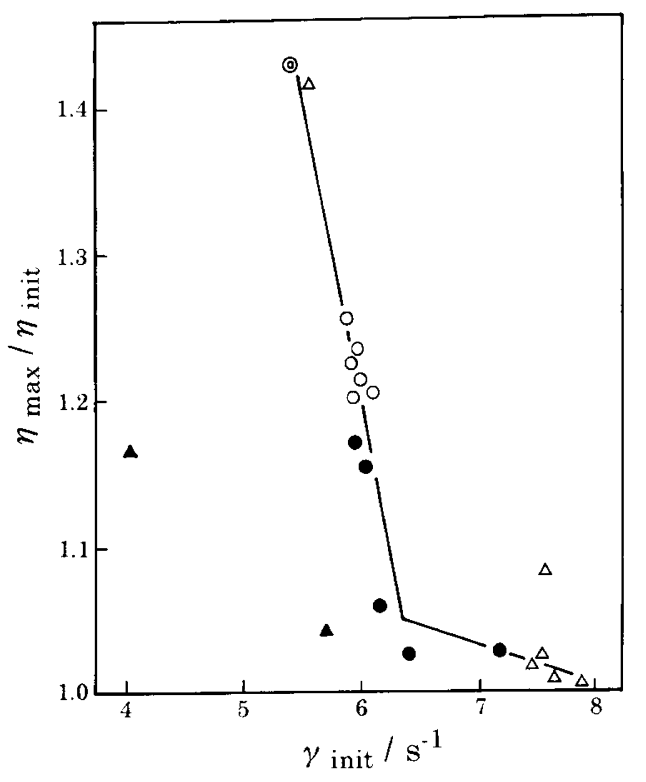

Figure 10. Rheopexy $\left(\eta_{\max } / \eta_{\text {init }}\right)$ as a function of $\gamma_{\text {init }}$ in the presence of additives : $\mathrm{NaCl}(\bigcirc), \mathrm{HCl}(\bigcirc)$, and urea $(\Delta$ and $\boldsymbol{\Delta}$ in the upturn region); (O) refers to the data without the additives.

value of $V_{\mathrm{e}}=6.45 \times 10^{-16} \mathrm{dL}$ was obtained.

The total volume which is occupied with all the equivalent sphere, $V_{\text {total }}$, is given as,

$$
V_{\text {total }}=N V_{\mathrm{e}}=\left(c N_{\mathrm{A}} / M\right) V_{\mathrm{e}}
$$

where $N$ and $N_{\mathrm{A}}$ are number of polymer molecules existing in $1 \mathrm{dL}$ of the solution and Avogadro's number, respectively. Consequently, the value of $V_{\text {total }}=34.3$ was obtained. It is considered, therefore, that at least $35 \%$ of space in the solution must be occupied by the domain of PMA molecules for the germination of rheopexy. It should be noticed here incidentally that the critical concentration depends on the initial rate of shear, $\gamma_{\text {init }}$.

In attempt to obtain further information on the nature of rheopexy, several kinds of substances were selected as the additives : hydrochloric acid and sodium hydroxide have effect on dissociation, sodium chloride on ionic atmosphere in the solution, and urea on hydrophobic bond of the PMA chains and on the water structure. The results represented in Figures 5 to 8 indicate that these additives affect considerably rheopexy of the PMA solution.

Roughly speaking, sodium hydroxide is distinguished in the action on rheopexy from the others, since its addition results only in a monotonous reduction of the ratio, $\eta_{\max } / \eta_{\text {init }}$, and in a progressive augmentation in $\eta_{\text {init }}$ (Figure 6). This behavior is explained simply in terms of the strong repulsive interaction between the increased carboxyl anions. That is to say, PMA molecules are not only compelled to occupy more expanded conformation, but also prevented from yielding some sort of intramolecular and intermolecular bonds, in particular, the latter of which would be regarded as the origin of rheopexy.

Addition of the other substances, on the contrary, leads first to a sudden decrease in $\eta_{\max } / \eta_{\text {init }}$ and then a gradual increase in the ratio above a given amount of addition; on this occation $\eta_{\text {init }}$ varies virtually in parallel with $\eta_{\max } / \eta_{\text {init }}$ (Figures 5,7 , and 8). A role of charges on 
the PMA chains may be unimportant in these cases, because even in the solution without any additive, only one per one thousand carboxyl groups dissociate. Furthermore, these additives have little effect (sodium chloride and urea) or a depressive action (hydrochloric acid) on dissociation of the carboxyl groups. It is somewhat suspected also that change in viscosity of the solution caused by the additives is a major factor for the alteration of the rheopectic behavior, for it is accompanied simultaneously by a change in the rate of shear. Hence it seems reasonable to consider that the cohesive force of the molecular clusters would be so weak that they are disintegrated readily at overgrowth and/or at high shear stress, for the same reason as discussed already on the aqueous solution. In such an anticipation, the ratio $\eta_{\max } /$ $\eta_{\text {init }}$ is plotted against the initial rate of shear $\gamma_{\text {init }}$ in Figure 10. Data for the systems of hydrochloric acid and sodium chloride are confined to those in the concentration range that the upturn of $\eta_{\max } / \eta_{\text {init }}$ does not yet start, since the solutions become unstable over this range. Irrespective of the additive species, as is to be expected, a correlation appears to exist between rheopexy and the rate of shear. It should be remarked that data for the solutions containing urea deviate markedly from a common curve in the upturn region. The influence of urea on the PMA solution is particularly interesting : in the range of concentration of urea examined here, the solutions settled are stable and do not give any indication of phase separation, which are different from the other two; further the concentration of urea showing the upturns of $\eta_{\text {init }}$ and $\eta_{\max } / \eta_{\text {init }}$ is interestingly in the region where proteins are denatured. $^{42}$

Although the present studies cannot cast much light into the molecular mechanism of rheopexy, it seems that the sole hydrogen bonding ability of PMA molecules is not a sufficient factor to induce rheopexy, based on the fact that the aqueous PAA solutions showed no rheopexy in this work. Since PMA differs from PAA only by existence of $\alpha$-methyl group, it may be concluded that the hydrophobic interaction between PMA molecules plays an important role in rheopexy, and that a particular balance between the hydrophilic bonding ability and the hydrophobic one of PMA molecules is an essential factor to induce rheopexy. This is also supported by the fact that urea affects rheopexy in the solution of PMA remarkably. Such an effect of urea has been explained by the consideration that urea affects both the intramolecular and intermolecular hydrophobic bonds of polymers through destruction of the iceberg structure of water surrounding the hydrophobic groups of the solute molecules. ${ }^{43}$

Acknowledgments. The authors are grateful to Prof. $K$. Iwata for the helpful suggestions and discussions and to Mr. M. Sasaki for some assistance for construction of the viscometer.

\section{REFERENCES}

1. J. Eliassaf, A. Silberberg, and A. Katchalsky, Nature, 176, 1119 (1955).

2. T. Matsuo, A. Peterlin, and D. T. Turner, J. Colloid Interface Sci., 24, 241 (1967).

3. A. Pavan, T. Matsuo, A. Peterlin, and D. T. Turner, J. Colloid
Interface Sci., 24, 273 (1967).

4. J. G. Watterson and M. C. Schaub, J. Colloid Interface Sci., 45, 280 (1973).

5. a) S. Ohoya, T. Matsuo, and M. Shioda, The 22nd Polymer Symposium of the Society of Polymer Science Japan, Tokyo, Nov., 1973, Abstr., p III -37. b) S. Ohoya and T. Matsuo, J. Colloid Interface Sci., 68, 593 (1979).

6. G. Marrucci, S. Bhargava, and S. L. Cooper, Macromolecules, 26, 6483 (1993).

7. D. Dupuis and C. Wolff, J. Rheol ., 37, 587 (1993).

8. D. Renoux, J. Selb, and F. Candau, Prog. Colloid Polym. Sci., 97, 213 (1994).

9. Y. Hu, S. Q. Wang, and A. M. Jamieson, Macromolecules, 28, $1847(1995)$

10. S. Biggs, J. Selb, and F. Candau, Langmuir, 8, 838 (1992).

11. Y. Hu, S. Q. Wang, and A. M. Jamieson, J. Colloid Interface Sci., 156, 31 (1993).

12. N. Willenbacher, J. Colloid Interface Sci., 182, 501 (1996).

13. D. Danino, Y. Talman, and R. Zana, Langmuir, 11, 1448 (1995).

14. J. W. Bender and N. J. Wagner, J. Colloid Interface Sci., 172, 171 (1995).

15. J. Castle, A. Merrington, and L. V. Woodcoock, Prog. Colloid Polym. Sci., 98, 111 (1995).

16. H. Verduin, B. J. deGans, and K. G. Dhont, Langmuir, 12 , 2947 (1996).

17. A. M. Liquori, G. Barone, V. Crescenzi, F. Quadrifoglio, and V. Vitagliano, J. Macromol. Chem., 1, 291 (1966).

18. Z. Priel and A. Silberberg, J. Polym. Sci., A-2, 8, 689 (1970).

19. J. C. Lyte and M. Mandel, J. Polym. Sci., A, 2, 1879 (1964).

20. M. Mandel, J. C. Lyte, and M. G. Stadhouder, J. Phys. Chem., 71, 603 (1967).

21. A. Silberberg and P. F. Mijnlieff, J. Polym. Sci., A-2, 8, 1089 (1970).

22. P. Dubin and U. P. Strauss, J. Phys. Chem., 77, 1427 (1973).

23. C. Braud, G. Muller, and E. Sélégny, Eur. Polym. J., 14, 479 (1978).

24. S. M. Towlson and P. V. Wright, Polymer, 27, 417 (1986).

25. B. Bednár, J. Trnéná, P. Svoboda, S. Bajda, V. Fidler, and K. Procházka, Macromolecules, 24, 2054 (1991).

26. M. Sakurai, T. Imai, F. Yamashita, K. Nakamura, and T. Komatsu, Polym. J., 25, 1247 (1993).

27. S. Kawaguchi, T. Takahashi, H. Tajima, Y. Hirose, and K. Ito, Polym. J., 28, 735 (1996).

28. J. Eliassaf, J. Polym. Sci., Polym. Lett. Ed., 3, 767 (1965).

29. G. Barone, V. Crescenzi, B. Pispisa, and F. Quadrifoglio, J. Macromol. Chem., 1, 761 (1966).

30. G. Barone, V. Crescenzi, A. M. Liquori, and F. Quadrifoglio, $J$. Phys. Chem., 71, 2341 (1967).

31. B. D. Ratner and I. F. Miller, J. Polym. Sci., A-1, 10, 2425 (1972).

32. J. Eliassaff and A. Silberberg, J. Polym. Sci., 41, 33 (1959).

33. K. Tsubakiyama, T. Matsuo, T. Sasaki, K. Yoshida, and K. Araki, J. Polym. Sci., Polym. Chem. Ed ., 17, 1829 (1979).

34. M. Kurata and Y. Tsunashima, in "Polymer Handbook", 4th Ed., J. Brandup, E. H. Immergut, and E. A. Grulke, Ed., John Wiley \& Sons Inc., New York, N. Y., 1999, pVI-1.

35. A. R. Sloniewsky, G. T. Evans, and P. Ander, J. Polym. Sci., A $-2,6,1555$ (1968).

36. G. C. Berry, J. Chem. Phys., 46, 1338 (1967).

37. S. Onogi, "Kagakushanotameno REOROJI", Kagakudojin, Kyoto, 1997, p 47.

38. K. Ono and K. Murakami, J. Polym. Sci., Polym. Lett. Ed., 15, 507 (1977).

39. P. J. Flory and T. G. Fox, Jr., J. Am. Chem. Soc., 73, 1904 (1951).

40. J. Eliassaf and A. Silberberg, Polymer, 3, 555 (1962).

41. R. M. Fuoss, J. Polym. Sci., 3, 603 (1948).

42. D. Eagland, in "Water, A Comprehensive Treatise", Vol. 2, F. Franks, Ed., Plenum Press, New York, N. Y., 1972, Chaps. 4 and 5 .

43. F. Franks, F. Franks, and D. S. Reid, in "Water, A Comprehensive Treatise", Vol. 2, F. Franks, Ed., Plenum Press, New York, N. Y., 1972, Chaps. 1 and 5. 